\title{
Synthesis of new bi(pyrazolo[1,5-a]pyrimidinyl)-7-one derivatives from dehydroacetic acid and its analogues as antibacterial agents
}

\author{
Ranjana Aggarwal, ${ }^{\text {a* }}$ Chinu Rani, ${ }^{a}$ Rajiv Kumar, ${ }^{a}$ Gaurav Garg, ${ }^{\text {b }}$ and Jitender Sharma ${ }^{b}$ \\ ${ }^{a}$ Department of Chemistry, Kurukshetra University, Kurukshetra-136119, India \\ ${ }^{b}$ Department of Microbiology, Kurukshetra University, Kurukshetra-136119, India \\ E-mail: ranjanaaggarwal67@gmail.com, ranjana67in@yahoo.com
}

\section{Dedicated to Prof. Rosa Maria Claramunt on the occasion of her 65th anniversary}

\begin{abstract}
Synthesis of some new bi(pyrazolo[1,5-a]pyrimidinyl)-7-ones $\mathbf{3}$ was accomplished by the reaction of 3- and/or 4-substituted 5-aminopyrazoles 2 with 3-acetyl-4-hydroxy-6-methyl2H-pyran-2-one (dehydroacetic acid, DHAA) 1a and its analogues 1b-c in refluxing ethanol. A tentative mechanism for the formation of $\mathbf{3}$ is proposed. These compounds were evaluated for their antibacterial activity against two Gram positive bacteria i.e., Bacillus subtilis (MTCC 8509) and Bacillus stearothermophilus (MTCC 8505) and two Gram negative bacteria i.e. Escherichia coli (MTCC 119) and Pseudomonas aeruginosa (MTCC 741) using agar well diffusion assay technique and minimum inhibitory concentration (MIC) method. One of the compound 3d displayed significant antibacterial activity.
\end{abstract}

Keywords: Dehydroacetic acid, bi(pyrazolo[1,5-a]pyrimidinyl)-7-ones, 5-aminopyrazoles antibacterial agents

\section{Introduction}

3-Acetyl-4-hydroxy-6-methyl-2H-pyran-2-one (dehydroacetic acid, DHAA) 1a, having several electrophilic sites, is susceptible to attack by nucleophilic reagents at carbonyl of the acetyl side chain at position-3, carbon terminating the conjugating carbon chain at 6-position, lactone carbonyl at 2-position and carbonyl at 4-position. ${ }^{1-2}$ Binucleophiles such as mono-substituted hydrazines and hydroxylamine are known to react with DHAA to give a wide variety of products, e.g. pyrazoles, bipyrazoles, bis-isoxazole etc., under different reaction conditions. ${ }^{3-6} 5$ Amino-1H-pyrazoles like mono-substituted hydrazines have been utilized as active synthons for synthesis of many heterocyclic compounds, e.g., pyrazolo[1,5-a]pyrimidines, pyrazolo[3,4$b$ ]pyridines, etc. ${ }^{7-11}$ Large differences in the nucleophilicity of the two nitrogens in 5-amino- $1 H$ pyrazoles, one as endo nitrogen of pyrazole ring and the other as amino group at 5-position, 
makes it an ideal binucleophile. Thus, the reaction of 5-aminopyrazoles with $\beta$-diketones may take place with the participation of a primary amino group in the first step and subsequent cyclization by the attack of ring nitrogen or vice versa to give regioisomeric pyrazolo[1,5a]pyrimidines. ${ }^{7,10-14}$

A literature survey reveals that the pyrazolo[1,5-a]pyrimidine skeleton has been applied in various therapeutic areas such as antitumor, antidiabetic, benzodiazepine receptor activities, etc. $^{15-16}$ Also, the pyrazolopyrimidines ${ }^{17}$ are selective inhibitor of cyclic 3',5'-adenosine monophosphate (cAMP) phosphodiesterases in vitro, and some of them possess anxiolytic properties comparable to those of benzodiazepines. ${ }^{18}$

We have a constant need for novel compounds or principles to combat bacteria that have become resistant to conventional antibacterials. Therefore, in continuation of our work on the synthesis of pyrazole moiety ${ }^{19-20}$ and fused heterocycles, ${ }^{7,11,21-24}$ we now have aim to determine antibacterial activity and to describe the regioselective synthesis of new fused bi(pyrazolo[1,5a]pyrimidinyl)-7-ones, by the reaction of DHAA and its analogues with 5-aminopyrazoles.

\section{Results and Discussion}

The reaction of 5-amino-4-phenyl-1 $H$-pyrazole $\mathbf{2 a}$, obtained by the formylation of benzylcyanide followed by the reaction with hydrazine hydrate, was initially carried out with an equimolar amount of DHAA 1a in refluxing ethanol for $6 \mathrm{~h}$ which afforded 5,5'-dimethyl-3,3'-diphenyl-4H[6,7']bi(pyrazolo[1,5-a]pyrimidinyl)-7-one 3a (Scheme 1). The yield of the reaction was very low as some DHAA was left unreacted, however, on using two equivalents of 5-aminopyrazole 2a there was a tremendous improvement in the yield of the reaction from $35 \%$ to $79 \%$ and the tlc showed disappearance of DHAA. Also, using a catalytic amount of acetic acid in ethanol resulted in the reduction of reaction time to $5 \mathrm{~h}$. The structure of the compound $\mathbf{3 a}$ was identified on the basis of the IR, ${ }^{1} \mathrm{H},{ }^{13} \mathrm{C}$ NMR and mass spectra. The IR spectrum of 3a showed absorption bands at 3472 and $1637 \mathrm{~cm}^{-1}$ due to $\mathrm{N}-\mathrm{H}$ and $\mathrm{C}=\mathrm{O}$ stretch, respectively. The ${ }^{1} \mathrm{H}$ NMR spectrum of $3 \mathbf{a}$ shows the presence of two singlets at $\delta 2.28$ and $2.72 \mathrm{ppm}$ due to the presence of two methyl protons at positions 5 and 5 ' respectively. Appearance of three sharp singlets each of one proton intensity at $\delta 6.89,7.99$ and $8.35 \mathrm{ppm}$ corresponding to bi(pyrazolopyrimidine) 6', 2 and $2^{\prime}-\mathrm{H}$ shows the formation of bi(pyrazolo[1,5-a]pyrimidine) nucleus. ${ }^{25-26}$ The ${ }^{1} \mathrm{H}$ NMR spectrum of $3 \mathbf{a}$ also showed one exchangeable proton at $\delta 13.05 \mathrm{ppm}$ due to NH present at position-4.

To generalize the reaction, different substituted bi(pyrazolo[1,5-a]pyrimidines) $\mathbf{3 b} \mathbf{b}-\mathbf{3 e}$ were prepared by reacting differently substituted 5-amino-4-phenyl- $1 H$-pyrazoles 2a-b with DHAA 1a and its analogues 1b-c in a ratio of 2:1 (Scheme 1). All the compounds were identified on the basis of the IR, ${ }^{1} \mathrm{H},{ }^{13} \mathrm{C}$ NMR and mass spectral data. In the ${ }^{1} \mathrm{H}$ NMR spectrum of $\mathbf{3 b}$, one of the methyl of DHAA is replaced by ethyl group and a typical pattern of triplet at $\delta 1.14 \mathrm{ppm}$ and quartet at $\delta 3.06 \mathrm{ppm}$ appeared along with the other signals as were obtained in the spectrum of 3a. Proton present at 6'-H disappears in $\mathbf{3 d}$ as this proton is replaced by bromine atom. Also, two 
singlets at about $\delta 7.99$ and $8.35 \mathrm{ppm}$, which appeared for two pyrazole ring 2 and 2'-H in 3a and $3 \mathbf{b}$, were replaced by that of two singlets of methyl protons between $\delta 2.39-2.51 \mathrm{ppm}$ and 2.55 $2.65 \mathrm{ppm}$ in 3c-3d for 2 and $2^{\prime}-\mathrm{CH}_{3}$, respectively. The ${ }^{13} \mathrm{C}$ NMR spectra of 3a-e also showed 12 signals for the basic skeleton along with other indispensable signals for the substituents, thus confirming the condensation of two moles of pyrazole moiety with one mole of DHAA, and its analogues.

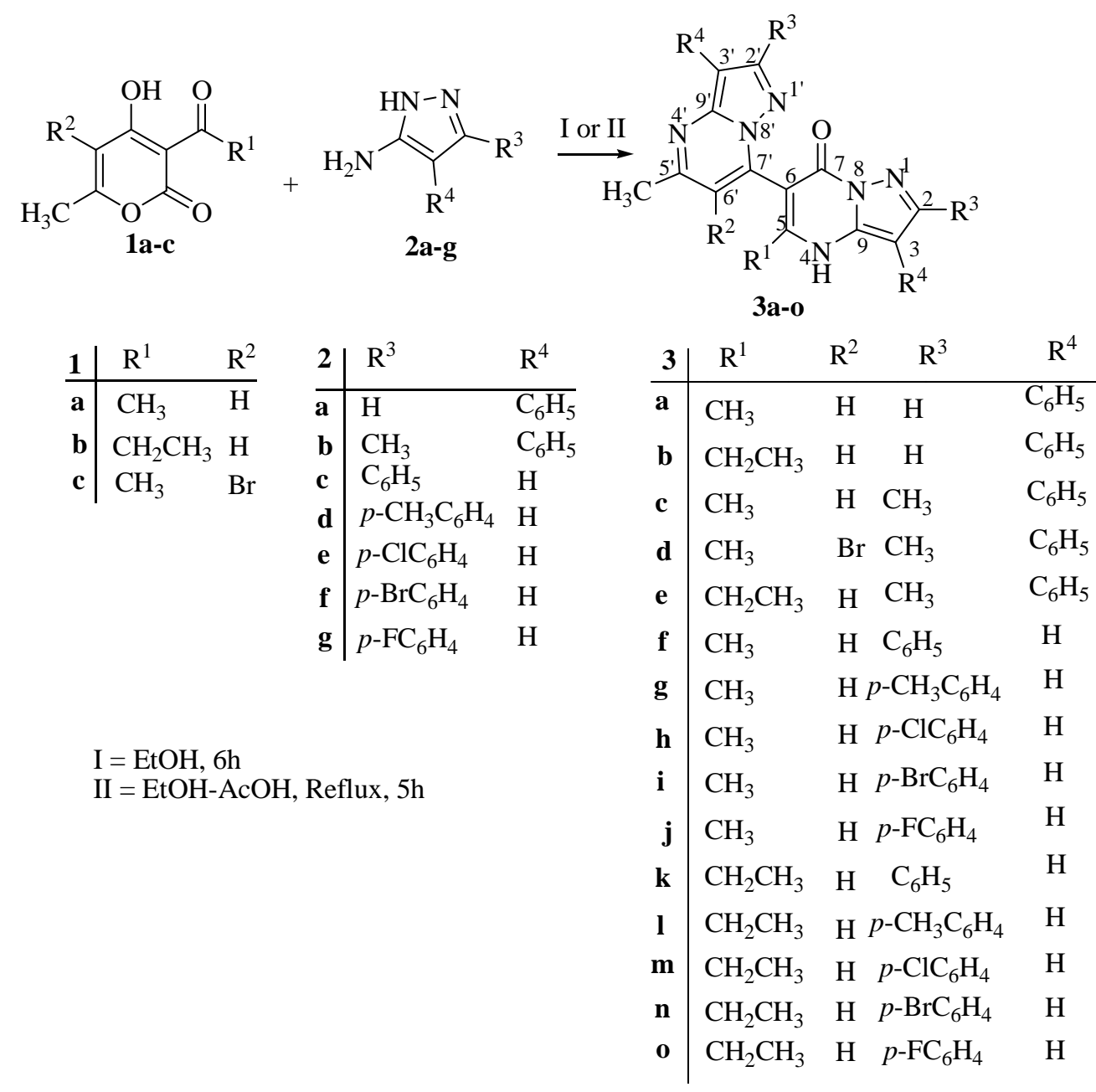

Scheme 1. Synthesis of bi(pyrazolo[1,5-a]pyrimidinyl)-7-ones 3a-o from 5-aminopyrazoles 2a-g and DHAA and its analogues 1a-c.

An extensive literature survey of the chemical behavior of $\mathrm{N}$-unsubstituted 5-amino- $1 \mathrm{H}$ pyrazoles 2 ring having vacant 4-position revealed that it may react with electrophiles such as $\beta$ diketones 4 to yield a mixture of structural isomers pyrazolo[1,5- $a$ ]pyrimidines $\mathbf{5}$ and pyrazolo[3,4-b]pyridines 6 (Scheme 2) depending upon the nature of substituent on $\beta$ diketones. ${ }^{27}$ Solvent also plays a crucial role in controlling the structure of the product as acetic 
acid leads to the formation of a mixture of pyrazolo[3,4- $b$ ]pyridine $\mathbf{6}$ and pyrazolo[1,5a]pyrimidine 5. Pyrazolopyrimidine $\mathbf{5}$ was formed as the only reaction product when DMSO was used as solvent whereas on boiling the reactants in ethanol in the presence of TEA yielded pyrazolopyridine 6 predominantly. ${ }^{28}$

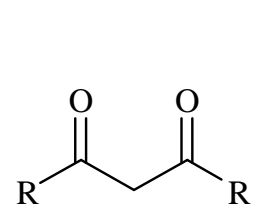

4

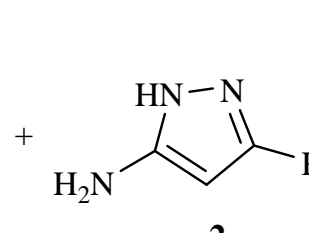

2

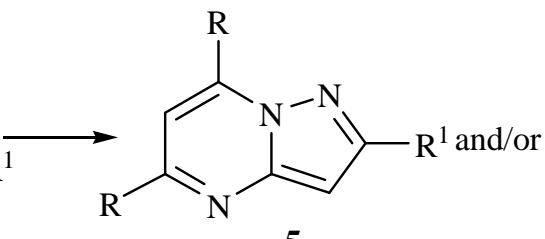

5

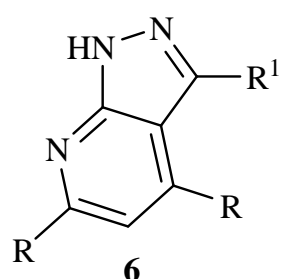

6

Scheme 2. Expected products from the reaction between $N$-unsubstituted 5-amino- $1 H$-pyrazoles 2 having vacant 4-position and $\beta$-diketones 4.

After having performed reactions with $\mathbf{2 a}$ and $\mathbf{2} \mathbf{b}$, it was thought of investigating the structure of products on treating 5-amino-3-aryl-1H-pyrazoles 2c-g (unsubstituted at position 4) with DHAA and its analogues 1a-b (Scheme 1). 5-Amino-3-aryl-1H-pyrazoles 2c-g, obtained from the condensation of $\alpha$-cyanoacetophenones and hydrazine hydrate in ethanol, on refluxing with DHAA and its analogues 1a-b resulted in the formation of single products which were characterized on the basis of their IR, ${ }^{1} \mathrm{H},{ }^{13} \mathrm{C}$ NMR and mass spectra as bi(pyrazolopyrimidinyl)7-ones 3f-o. No trace of pyrazolo[3,4- $b]$ pyridine was detected in the tlc and the NMR of the crude reaction mixture.

The ${ }^{1} \mathrm{H}$ NMR spectra of bi(pyrazolopyrimidines) 3f-o were almost identical with those of bi(pyrazolopyrimidines) 3a-e. One important difference is that the spectra showed three singlets of one proton intensity each between $\delta$ 6.71-6.82 ppm, 7.02-7.09 ppm and 7.08-7.19 ppm corresponding to 6', 3 and 3'-H, respectively, showing the presence of two pyrazolopyrimidine nuclei having a vacant 3 -position. The ${ }^{1} \mathrm{H}$ NMR spectra of $\mathbf{3 f - o}$ displayed only one exchangeable proton at about $\delta 13.05 \mathrm{ppm}$ due to $\mathrm{NH}$ present at position-4. Had the structure been pyrazolopyridine then the signals for 3 and 3'-H would not have appeared rather exchangeable signals for three NH protons would have been observed in the NMR and IR spectra.

A plausible mechanism leading to the formation of bi(pyrazolo[1,5-a]pyrimidinyl)-7-ones 3a-o is depicted in Scheme 3. An amino group of 5-aminopyrazole 2 attacks on carbonyl of acetyl side chain of $\mathbf{1}$ to generate a Schiff's base intermediate $\mathbf{1}^{\prime}$, followed by the subsequent nucleophilic attack of the endo nitrogen on the lactone carbonyl of DHAA to provide a pyrazolo[1,5- $a$ ]pyrimidinyl- $\beta$-diketone 7 through an intramolecular pyran ring opening (Scheme 3). This mechanism is supported by the fact that 5-aminopyrazoles react with $\beta$-ketoesters to produce pyrazolo[1,5-a]pyrimidinyl-7-ones as the single product involving the first attack of primary amino group on the keto group and subsequent attack of endo $\mathrm{N}$ on ester group. ${ }^{17,29-30}$ Efforts were made to isolate pyrazolo[1,5-a]pyrimidinyl- $\beta$-diketone 7 by performing the reaction at room temperature or in the cold or by adding the solution of 5-aminopyrazole $\mathbf{2 b}$ drop wise to the solution of DHAA 1 or by using two equivalent of DHAA 1 and one equivalent of 5- 
aminopyrazole $\mathbf{2 b}$ but the reaction did not stop at this level as the resulting $\beta$-diketones $\mathbf{7}$ has greater reactivity towards nucleophiles and the second mole of 5-aminopyrazole 2 reacted further with the $\beta$-diketone 7 to yield the bi(pyrazolo[1,5-a]pyrimidinyl)-7-ones 3 .

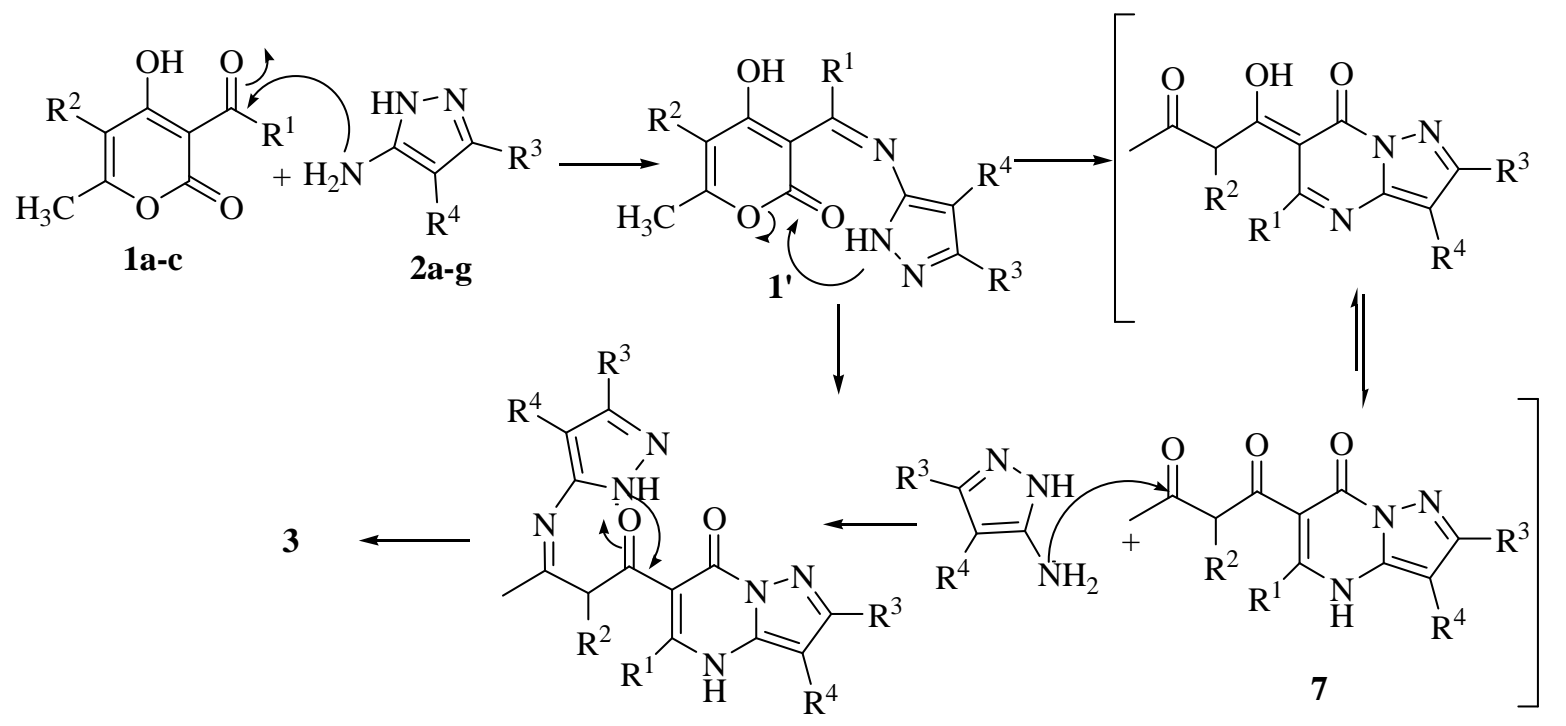

Scheme 3. Plausible mechanism leading to the formation of bi(pyrazolo[1,5-a]pyrimidinyl)-7ones 3a-o.

\section{In vitro antibacterial assay}

Primary screening. The antibacterial activities of these compounds, 5'-methyl-4H[6,7']bi(pyrazolo[1,5-a]pyrimidinyl)-7-ones 3a-o were evaluated against two Gram-positive bacteria i.e. Bacillus subtilis (MTCC 8509) and Bacillus stearothermophilus (MTCC 8505) and two Gram-negative bacteria i.e. Escherichia coli (MTCC 119) and Pseudomonas aeruginosa (MTCC 741) at three different concentrations i.e. 10, 50 and $100 \mu \mathrm{g} / \mathrm{mL}$ (Table 1) using agar well diffusion assay technique ${ }^{31-32}$ and minimum inhibitory concentration (MIC) method (Table 2).

The antimicrobial activities of the compounds were compared against the standard drugs, ampicillin, chloramphenicol $(25 \mu \mathrm{g} / \mathrm{mL})$ (+ve control) and DMSO (-ve control). These tests were performed in triplicate and the mean of diameter of zone of growth inhibition was taken. 
Table 1. In vitro antibacterial activity of compounds 3a-o by using well diffusion method

\begin{tabular}{|c|c|c|c|c|c|}
\hline \multirow[t]{2}{*}{ Compound } & \multirow{2}{*}{$\begin{array}{l}\text { Concentration } \\
(\mu \mathrm{g} / \mathrm{mL})\end{array}$} & \multicolumn{4}{|c|}{ Diameter of zone of growth inhibition (mm) } \\
\hline & & $\begin{array}{c}\text { B. } \\
\text { subtilis }\end{array}$ & $\begin{array}{c}\text { B. stearo- } \\
\text { thermophilus }\end{array}$ & $\begin{array}{c}E . \\
\text { coli }\end{array}$ & $\begin{array}{l}\text { P. aeru- } \\
\text { ginosa }\end{array}$ \\
\hline \multirow[t]{3}{*}{ 3a } & 10 & - & - & - & - \\
\hline & 50 & - & - & - & - \\
\hline & 100 & - & - & 13 & 12 \\
\hline \multirow[t]{3}{*}{$\mathbf{3 b}$} & 10 & 09 & - & - & - \\
\hline & 50 & 21 & 19 & 13 & 16 \\
\hline & 100 & 49 & 36 & 29 & 31 \\
\hline \multirow[t]{3}{*}{$3 c$} & 10 & - & 10 & 11 & 09 \\
\hline & 50 & 12 & 23 & 24 & 25 \\
\hline & 100 & 31 & 38 & 46 & 43 \\
\hline \multirow[t]{3}{*}{ 3d } & 10 & 16 & 13 & 13 & 17 \\
\hline & 50 & 31 & 29 & 28 & 30 \\
\hline & 100 & 65 & 57 & 53 & 59 \\
\hline \multirow[t]{3}{*}{$3 \mathbf{e}$} & 10 & - & - & - & - \\
\hline & 50 & 09 & - & 16 & 17 \\
\hline & 100 & 25 & 23 & 34 & 39 \\
\hline \multirow[t]{3}{*}{ 3f } & 10 & - & - & - & - \\
\hline & 50 & - & - & - & - \\
\hline & 100 & 10 & 11 & 13 & - \\
\hline \multirow[t]{3}{*}{$3 g$} & 10 & - & - & - & - \\
\hline & 50 & - & - & - & - \\
\hline & 100 & - & - & - & - \\
\hline \multirow[t]{3}{*}{$3 h$} & 10 & - & - & - & - \\
\hline & 50 & - & - & - & - \\
\hline & 100 & 11 & 14 & 12 & - \\
\hline \multirow[t]{3}{*}{$3 \mathbf{i}$} & 10 & - & - & - & - \\
\hline & 50 & - & - & - & - \\
\hline & 100 & - & 13 & 11 & 10 \\
\hline \multirow[t]{3}{*}{$3 \mathbf{j}$} & 10 & - & - & - & - \\
\hline & 50 & - & - & - & - \\
\hline & 100 & 10 & 12 & 13 & 12 \\
\hline \multirow[t]{3}{*}{ 3k } & 10 & - & - & - & - \\
\hline & 50 & - & 11 & 10 & - \\
\hline & 100 & 10 & 16 & 14 & 09 \\
\hline \multirow[t]{3}{*}{31} & 10 & - & - & - & - \\
\hline & 50 & - & - & - & - \\
\hline & 100 & - & - & - & - \\
\hline
\end{tabular}


Tabel 1 (continued)

\begin{tabular}{cccccc}
\hline $3 \mathbf{m}$ & 10 & - & - & - & - \\
& 50 & - & - & - & - \\
$3 \mathbf{3 n}$ & 100 & - & 14 & 13 & - \\
& 10 & - & - & - & - \\
30 & 50 & - & - & - & - \\
& 100 & 10 & 12 & 11 & - \\
& 10 & - & - & - & - \\
Chloramphenicol & 50 & - & - & - & - \\
Ampicillin $^{\mathrm{b}}$ & 100 & 11 & 13 & 10 & 10 \\
\hline
\end{tabular}

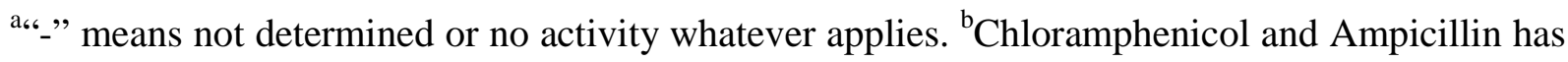
been used as a standard drugs.

Table 2. Minimum inhibitory concentration $\left(\mathrm{MIC}^{*}\right)(\mu \mathrm{g} / \mathrm{mL})$ of test compounds

\begin{tabular}{ccccc}
\hline Compounds & B. subtilis & B. stearothermophilus & E. coli & P. aeruginosa \\
\hline 3a & $>200$ & $>200$ & 100 & 100 \\
3b & 6.25 & 12.25 & 25 & 12.25 \\
$\mathbf{3 c}$ & 25 & 6.25 & 6.25 & 6.25 \\
$\mathbf{3 d}$ & 3.12 & 6.25 & 6.25 & 3.12 \\
$\mathbf{3 e}$ & 50 & 100 & 12.25 & 12.25 \\
$\mathbf{3 f}$ & 100 & 100 & 100 & $>200$ \\
$\mathbf{3 g}$ & $>200$ & $>200$ & $>200$ & $>200$ \\
$\mathbf{3 h}$ & 100 & 100 & 100 & $>200$ \\
$\mathbf{3 i}$ & 200 & 100 & 100 & 100 \\
$\mathbf{3 j}$ & 100 & 100 & 100 & 100 \\
$\mathbf{3 k}$ & 100 & 50 & 50 & 100 \\
$\mathbf{3 l}$ & $>200$ & $>200$ & $>200$ & $>200$ \\
$\mathbf{3 m}$ & $>200$ & 100 & 100 & $>200$ \\
$\mathbf{3 n}$ & 100 & 100 & 100 & $>200$ \\
$\mathbf{3 0}$ & 100 & 100 & 100 & 100 \\
Chloramphenicol & 0.19 & $<0.19$ & $<0.19$ & $<0.19$ \\
Ampicillin & $<0.19$ & $<0.19$ & $<0.19$ & $<0.19$ \\
\hline
\end{tabular}

*Means of triplicates.

From the Table 1, it may be concluded that the compounds bearing phenyl substituent at position-3 of pyrazolopyrimidine ring i.e. 3,3'-diphenyl-4H-[6,7']bi(pyrazolo[1,5-a]pyrimidinyl)7-ones 3a-d showed good result as compared to compounds having vacant 3-position 3f-o. More 
importantly, compound 3d bearing bromine at position-6' exhibited the maximum zone of inhibition followed by that for compounds $\mathbf{3 c}$ and $\mathbf{3 b}$ at concentration of 100,50 and $10 \mu \mathrm{g} / \mathrm{mL}$ against both Gram-positive and Gram-negative bacteria as compared to other compounds (3a, 3e-o). Compounds $3 \mathrm{~g}$ and $\mathbf{3 l}$ having p-methylphenyl at 2 and 2'-position showed no zone of inhibition at concentrations $10,50,100 \mu \mathrm{g} / \mathrm{mL}$. The antibacterial activity of these compounds was also compared with two commercially available antibiotics namely Chloramphenicol and Ampicillin. It is clear from the Table $\mathbf{1}$ that the compound $\mathbf{3 d}$ has the zone of inhibition compared to the standard antibiotic Chloramphenicol when used at the concentration of 25 $\mu \mathrm{g} / \mathrm{mL}$ whereas no compound is as effective as the Ampicillin when used at the concentration of $25 \mu \mathrm{g} / \mathrm{mL}$.

In the whole series, the MIC value of various synthesized compounds was evaluated between 200-0.19 $\mu \mathrm{g} / \mathrm{mL}$ against Gram-positive and Gram-negative bacteria (Table 2). The compound 3d was found to be the most effective against all Gram-positive and Gram-negative bacteria having the lowest MIC value of $3.12 \mu \mathrm{g} / \mathrm{mL}$ against $B$. subtilis and $P$. aeruginosa, $6.25 \mu \mathrm{g} / \mathrm{mL}$ against $B$. stearothermophilus and E. coli. Compound 3c showed MIC value of $6.25 \mu \mathrm{g} / \mathrm{mL}$ against $B$. stearothermophilus, $P$. aeruginosa and $E$. coli while $25 \mu \mathrm{g} / \mathrm{mL}$ against $B$. subtilis comparable with commercial antibiotics Chloramphenicol and Ampicillin which showed MIC value of $<0.19$ and $0.19 \mu \mathrm{g} / \mathrm{mL}$ against all the bacteria. Whereas the compounds $\mathbf{3 g}$ and $\mathbf{3 l}$ are having the MIC value greater than $200 \mu \mathrm{g} / \mathrm{mL}$.

\section{Conclusions}

A series of 5'-methyl-4H-[6,7']bi(pyrazolo[1,5-a]pyrimidinyl)-7ones 3a-o were synthesized from the reaction of substituted 5-amino- $1 H$-pyrazoles with DHAA and its analogues and were evaluated for their antibacterial activity against both Gram-positive and Gram-negative bacteria. Treatment of 5-aminopyrazoles with or without a substituent at position-4 with DHAA and its analogues afforded single compound having two prazolopyrimidine nuclei. Compounds bearing phenyl substituent at position-3 of pyrazolopyrimidine ring 3a-d showed good result as compared to compounds having vacant 3-position 3f-o. It is interesting to note that compound 3d displayed significant antibacterial activity compared to the standard antibiotic Chloramphenicol.

\section{Experimental Section}

General. The IR spectra of the compounds were recorded on Buck Scientific IR M-500 spectrophotometer using $\mathrm{KBr}$ pellets $\left(v_{\max }\right.$ in $\left.\mathrm{cm}^{-1}\right),{ }^{1} \mathrm{H}$ and ${ }^{13} \mathrm{C} \mathrm{NMR}$ spectra were obtained on a Bruker instrument at 300 and $75 \mathrm{MHz}$, respectively, chemical shifts are expressed in $\delta$-scale downfield from TMS as an internal standard. Elemental analysis was performed at RSIC, Lucknow, India. High resolution mass spectra (HRMS) were measured in EI mode on a Kratos 
MS-50 spectrometer, Mass Spectrometry Facility, University of California, San Francisco, USA. The ${ }^{1} \mathrm{H}$ NMR signals of the aromatic protons of substituents $\mathrm{R}^{3}$ (at positions 2 and $2^{\prime}$ compounds 3g-o), are marked with the sign ".

5,5'-Dimethyl-3,3'-diphenyl-4H-[6,7']bi(pyrazolo[1,5-a]pyrimidinyl)-7-one $\quad$ (3a). To an ethanolic solution of 5-amino-4-phenyl-1 $H$-pyrazole $\mathbf{2 a}(0.318 \mathrm{~g}, 2 \mathrm{mmol})$ was added 3 -acetyl-4hydroxy-6-methyl-2H-pyran-2-one 1a $(0.168 \mathrm{~g}, 1 \mathrm{mmol})$ and refluxed for $6 \mathrm{hr}$. The reaction was monitored by TLC. After completion of the reaction, solvent was distilled under reduced pressure. Solid thus obtained was washed with little cold ethanol and recrystallized from ethanol to give 3a: yield 79\%, mp 213-15 ${ }^{\circ} \mathrm{C}$; IR $\left(v_{\max }, \mathrm{cm}^{-1}\right): 3463,1631 .{ }^{1} \mathrm{H}$ NMR $\left(\mathrm{CDCl}_{3}+\mathrm{DMSO}\right) \delta_{\mathrm{H}}$ : $2.28\left(\mathrm{~s}, 3 \mathrm{H}, 5-\mathrm{CH}_{3}\right), 2.72\left(\mathrm{~s}, 3 \mathrm{H}, 5{ }^{\prime}-\mathrm{CH}_{3}\right), 6.89\left(\mathrm{~s}, 1 \mathrm{H}, 66^{\prime}-\mathrm{H}\right), 7.23-7.26(\mathrm{~m}, 1 \mathrm{H}, \mathrm{Ph}), 7.34-7.38$ (m, 1H, Ph), 7.42-7.46 (m, 2H, Ph), 7.46-7.50 (m, 2H, Ph), 7.55-7.56 (m, 2H, Ph), 7.99 (s, 1H, 2$\mathrm{H}), 8.08-8.10(\mathrm{~m}, 2 \mathrm{H}, \mathrm{Ph}), 8.35\left(\mathrm{~s}, 1 \mathrm{H}, 2^{\prime}-\mathrm{H}\right), 13.05\left(\mathrm{~s}, 1 \mathrm{H}, \mathrm{NH}\right.$, exchangeable with $\left.\mathrm{D}_{2} \mathrm{O}\right) .{ }^{13} \mathrm{C}$ NMR $\left(\mathrm{CDCl}_{3}+\mathrm{DMSO}\right) \delta_{\mathrm{C}}: 18.01,24.95,94.62,99.21,109.64,112.82,125.91,126.10,127.06$, 128.19, 128.56, 128.85, 129.98, 132.19, 134.38, 136.58, 140.11, 141.95, 145.13, 151.45, 154.54, 158.77. MS (m/z): Calcd 433.17, found $433.17\left([\mathrm{M}+\mathrm{H}]^{+}\right)$; Anal. Calcd for $\mathrm{C}_{26} \mathrm{H}_{20} \mathrm{~N}_{6} \mathrm{O}: \mathrm{C}, 72.21$; H, 4.66; N, 19.43\%. Found: C, 72.19; H, 4.62; N, 19.25\%.

This reaction procedure without modification was used for the synthesis of all other compounds 3b-o.

5-Ethyl-5'-methyl-3,3'-diphenyl-4H-[6,7']bi(pyrazolo[1,5-a]pyrimidinyl)-7-one (3b). Yield 87\%, mp 195-98 ${ }^{\circ} \mathrm{C}$; IR $\left(v_{\max }, \mathrm{cm}^{-1}\right): 3462,1629 ;{ }^{1} \mathrm{H}$ NMR $\left(\mathrm{CDCl}_{3}+\mathrm{DMSO}\right) \delta_{\mathrm{H}}: 1.14(\mathrm{t}, J$ $\left.7.56 \mathrm{~Hz}, 3 \mathrm{H}, \mathrm{CH}_{3}\right), 2.72\left(\mathrm{~s}, 3 \mathrm{H}, 5^{\prime}-\mathrm{CH}_{3}\right), 3.08$ (q, J 7.56Hz, 2H, $\left.\mathrm{CH}_{2}\right), 6.86(\mathrm{~s}, 1 \mathrm{H}, 6$ '-H), $7.23-$ 7.27 (m, 1H, Ph), 7.34-7.38 (m, 1H, Ph), 7.42-7.46 (m, 2H, Ph), 7.47-7.51 (m, 2H, Ph), 7.54-7.56 (m, 2H, Ph), 7.99 (s, 1H, 2-H), 8.08-8.10 (m, 2H, Ph), 8.34 (s, 1H, 2'-H) , 13.08 (s, 1H, NH, exchangeable with $\left.\mathrm{D}_{2} \mathrm{O}\right) .{ }^{13} \mathrm{C}$ NMR $\left(\mathrm{CDCl}_{3}+\mathrm{DMSO}\right) \delta_{\mathrm{C}}: 13.69,24.88,25.32,94.53,99.12$, 109.48, 112.67, 125.83, 125.98, 126.97, 128.10, 128.49, 128.77, 129.87, 132.09, 140.14, 141.89, 143.21, 145.03, 151.31, 155.27, 156.15, 158.74. MS $(\mathrm{m} / \mathrm{z})$ : Calcd 447.18, found 447.19 $\left([\mathrm{M}+\mathrm{H}]^{+}\right)$; Anal. Calcd for $\mathrm{C}_{27} \mathrm{H}_{22} \mathrm{~N}_{6} \mathrm{O}: \mathrm{C}, 72.63 ; \mathrm{H}, 4.97 ; \mathrm{N}, 18.82 \%$. Found: $\mathrm{C}, 72.51 ; \mathrm{H}, 4.86$; $\mathrm{N}, 18.54 \%$.

2,5,2',5'-Tetramethyl-3,3'-diphenyl-4H-[6,7']bi(pyrazolo[1,5-a]pyrimidinyl)-7-one (3c). Yield 75\%, mp 228-30 ${ }^{\circ} \mathrm{C}$; IR $\left(v_{\max }, \mathrm{cm}^{-1}\right): 3458,1632 ;{ }^{1} \mathrm{H}$ NMR $\left(\mathrm{CDCl}_{3}+\mathrm{DMSO}\right) \delta_{\mathrm{H}}: 2.22(\mathrm{~s}$, $\left.3 \mathrm{H}, 5-\mathrm{CH}_{3}\right), 2.40\left(\mathrm{~s}, 3 \mathrm{H}, 2-\mathrm{CH}_{3}\right), 2.55\left(\mathrm{~s}, 3 \mathrm{H}, 2^{\prime}-\mathrm{CH}_{3}\right), 2.64\left(\mathrm{~s}, 3 \mathrm{H}, 5\right.$ '- $\left.\mathrm{CH}_{3}\right), 6.78(\mathrm{~s}, 1 \mathrm{H}, 6$ '- $\mathrm{H})$, 7.26-7.31 (m, 1H, Ph), 7.37-7.41 (m, 1H, Ph), 7.44-7.51 (m, 6H, Ph), 7.72-7.74 (m, 2H, Ph), 13.07 (s, $1 \mathrm{H}, \mathrm{NH}$, exchangeable with $\left.\mathrm{D}_{2} \mathrm{O}\right) .{ }^{13} \mathrm{C} \mathrm{NMR}\left(\mathrm{CDCl}_{3}+\mathrm{DMSO}\right) \delta_{\mathrm{C}}: 12.98,14.17,17.92$, 24.68, 100.05, 104.51, 108.26, 112.16, 125.91, 127.13, 128.24, 128.66, 128.76, 129.76, 130.60, $132.40,138.12,139.58,146.13,150.88,151.51,153.58,154.67,158.26 . \mathrm{MS}(\mathrm{m} / \mathrm{z}):$ Calcd 461.20, found $461.53\left([\mathrm{M}+\mathrm{H}]^{+}\right)$; Anal. Calcd for $\mathrm{C}_{28} \mathrm{H}_{24} \mathrm{~N}_{6} \mathrm{O}: \mathrm{C}, 73.03 ; \mathrm{H}, 5.25 ; \mathrm{N}, 18.25 \%$. Found: C, 73.21; H, 5.66; N, 18.16\%.

2,5,2',5'-Tetramethyl-3,3'-diphenyl-6'-bromo-4H-[6,7']bi(pyrazolo[1,5-a]pyrimidinyl)-7-one (3d). Yield 80\%, mp $240{ }^{\circ} \mathrm{C}(\mathrm{d})$; IR $\left(v_{\max }, \mathrm{cm}^{-1}\right): 3471,1640 ;{ }^{1} \mathrm{H} \mathrm{NMR}\left(\mathrm{CDCl}_{3}+\mathrm{DMSO}\right) \delta_{\mathrm{H}}: 2.36$ 
(s, 3H, 5- $\left.\mathrm{CH}_{3}\right), 2.51\left(\mathrm{~s}, 3 \mathrm{H}, 2-\mathrm{CH}_{3}\right), 2.65\left(\mathrm{~s}, 3 \mathrm{H}, 2^{\prime}-\mathrm{CH}_{3}\right), 2.83\left(\mathrm{~s}, 3 \mathrm{H}, 5\right.$ '- $\left.-\mathrm{CH}_{3}\right), 7.28-7.38(\mathrm{~m}, 4 \mathrm{H}$, $\mathrm{Ph})$, 7.42-7.49 (m, 4H, Ph), 7.71-7.73 (m, 2H, Ph), $13.05\left(\mathrm{~s}, 1 \mathrm{H}, \mathrm{NH}\right.$, exchangeable with $\left.\mathrm{D}_{2} \mathrm{O}\right)$. ${ }^{13} \mathrm{C} \mathrm{NMR}\left(\mathrm{CDCl}_{3}+\mathrm{DMSO}\right) \delta_{\mathrm{C}}: 10.50,14.38,14.61,21.91,100.11,106.20,108.52,116.34$, $126.62,126.80,128.38,128.61,128.83,128.92$, 131.20, 132.99, 138.32, 139.44, 147.36, 150.81, 151.51, 154.41, 154.77, 157.57. MS (m/z): Calcd 541.11, $539.11(1: 1)$, found 539.11, 541.11 $(1: 1)\left([\mathrm{M}+\mathrm{H}]{ }^{+} /[\mathrm{M}+\mathrm{H}+2]^{+}\right)$; Anal. Calcd for $\mathrm{C}_{28} \mathrm{H}_{23} \mathrm{BrN}_{6} \mathrm{O}: \mathrm{C}, 62.34 ; \mathrm{H}, 4.30 ; \mathrm{N}, 15.58 \%$. Found: C, 62.22; H, 4.22; N, 15.21\%.

5-Ethyl-2,2',5'-trimethyl-3,3'-diphenyl-4H-[6,7']bi(pyrazolo[1,5-a]pyrimidinyl)-7-one (3e). Yield $81 \%, \mathrm{mp} 230{ }^{\circ} \mathrm{C}$; IR $\left(v_{\max }, \mathrm{cm}^{-1}\right): 3465,1635 ;{ }^{1} \mathrm{H}$ NMR $\left(\mathrm{CDCl}_{3}+\mathrm{DMSO}\right) \delta_{\mathrm{H}}: 1.14(\mathrm{t}, J$ $\left.7.16 \mathrm{~Hz}, 3 \mathrm{H}, \mathrm{CH}_{3}\right), 2.39$ (s, 3H, 2- $\left.\mathrm{CH}_{3}\right), 2.53\left(\mathrm{~s}, 3 \mathrm{H}, 2{ }^{\prime}-\mathrm{CH}_{3}\right), 2.63$ (s, 3H, 5'- $\left.\mathrm{CH}_{3}\right), 2.89$ (q, J $\left.7.16 \mathrm{~Hz}, 2 \mathrm{H}, \mathrm{CH}_{2}\right), 6.75$ (s, 1H, 6'-H), 7.26-7.30 (m, 1H, Ph), 7.36-7.40 (m, 1H, Ph), 7.44-7.48 (m, 4H, Ph), 7.51-7.53 (m, 2H, Ph), 7.72-7.75 (m, 2H, Ph), 13.06 (s, 1H, NH, exchangeable with $\left.\mathrm{D}_{2} \mathrm{O}\right) .{ }^{13} \mathrm{C} \mathrm{NMR}\left(\mathrm{CDCl}_{3}+\mathrm{DMSO}\right) \delta_{\mathrm{C}}: 12.95,13.67,14.14,24.69,25.31,99.48,104.49,108.29$, $112.11,125.89,127.09,128.24,128.63,128.74,129.77,130.64,132.42,138.13,139.58,146.13$, 151.52, 151.57, 154.81, 155.66, 158.22. MS (m/z): Calcd 475.22, found 475.23 $\left([\mathrm{M}+\mathrm{H}]^{+}\right)$; Anal. Calcd for $\mathrm{C}_{29} \mathrm{H}_{26} \mathrm{~N}_{6} \mathrm{O}: \mathrm{C}, 73.40 ; \mathrm{H}, 5.52 ; \mathrm{N}, 17.71 \%$. Found: $\mathrm{C}, 73.23 ; \mathrm{H}, 5.62 ; \mathrm{N}, 17.53 \%$.

5,5'-Dimethyl-2,2'-diphenyl-4H-[6,7']bi(pyrazolo[1,5-a]pyrimidinyl)-7-one (3f). Yield 82\%, $\mathrm{mp}>330{ }^{\circ} \mathrm{C}$; IR $\left(v_{\max }, \mathrm{cm}^{-1}\right): 3063,1744,1705,1620,1551,1520,1458 ;{ }^{1} \mathrm{H}$ NMR $\left(\mathrm{DMSO}, \mathrm{d}^{6}\right)$ $\delta_{\mathrm{H}}: 2.22\left(\mathrm{~s}, 3 \mathrm{H}, 5-\mathrm{CH}_{3}\right), 2.60\left(\mathrm{~s}, 3 \mathrm{H}, 5^{\prime}-\mathrm{CH}_{3}\right), 6.79\left(\mathrm{~s}, 1 \mathrm{H}, 66^{\prime}-\mathrm{H}\right), 7.06(\mathrm{~s}, 1 \mathrm{H}, 3-\mathrm{H}), 7.15(\mathrm{~s}, 1 \mathrm{H}$, 3'-H), 7.34-7.53 (m, 6H, 2Ph-3", 4", 5"-H), 7.92 (d, 2 H, J 6.9 Hz, Ph-2", 6"-H), 8.04 (d, 2H, J $6.9 \mathrm{~Hz}, \mathrm{Ph}-2 ", 6$ "-H), 13.03 (s, 1H, NH, exchangeable with $\left.\mathrm{D}_{2} \mathrm{O}\right) .{ }^{13} \mathrm{C}$ NMR (DMSO, d $\left.\mathrm{d}^{6}\right) \delta_{\mathrm{C}}$ : $18.20,24.56,87.06,92.84,99.97,113.14,126.61,126.71,129.28,129.36,129.77,132.31$, 132.89, 140.46, 149.91, 154.47, 154.98, 159.40. MS $(\mathrm{m} / \mathrm{z})$ : Calcd 433.17, found 433.20 $\left([\mathrm{M}+\mathrm{H}]^{+}\right)$; Anal. Calcd. for $\mathrm{C}_{26} \mathrm{H}_{20} \mathrm{~N}_{6} \mathrm{O}$ : C, 72.21; H, 4.66; N, 19.43\%. Found: C, 72.18; H, 4.70; $\mathrm{N}, 19.40 \%$.

5,5'-Dimethyl-2,2'-di( $p$-methylphenyl)-4H-[6,7']bi(pyrazolo[1,5-a]pyrimidinyl)-7-one (3g). Yield 85\%, mp $280{ }^{\circ} \mathrm{C}(\mathrm{d})$; IR $\left(v_{\max }, \mathrm{cm}^{-1}\right): 3479,3240,3132,3063,2916,1659,1620,1558$, 1497, 1466; ${ }^{1} \mathrm{H}$ NMR (DMSO, d ${ }^{6}$ ) $\delta_{\mathrm{H}}: 2.20$ (s, 3H, 5- $\mathrm{CH}_{3}$ ), 2.32 (s, 3H, $\mathrm{CH}_{3}-\mathrm{Ph}$ ), 2.37 (s, 3H, $\left.\mathrm{CH}_{3}-\mathrm{Ph}\right), 2.59$ (s, 3H, 5'-CH $), 6.73$ (s, 1H, 6'-H), 7.02 (s, 1H, 3-H), 7.08 (s, 1H, 3'-H), 7.23 (d, 2 H, J 7.8 Hz, Ph-2", 6"-H), 7.30 (d, 2H, J 7.8 Hz, Ph-2", 6"-H), 7.81 (d, 2H, J 7.8 Hz, Ph-3", 5"$\mathrm{H}), 7.92(\mathrm{~d}, 2 \mathrm{H}, J 7.8 \mathrm{~Hz}, \mathrm{Ph}-3 ", 5 "-\mathrm{H}), 13.02\left(\mathrm{~s}, 1 \mathrm{H}, \mathrm{NH}\right.$, exchangeable with $\left.\mathrm{D}_{2} \mathrm{O}\right) .{ }^{13} \mathrm{C}$ NMR $\left(\mathrm{DMSO}, \mathrm{d}^{6}\right) \delta_{\mathrm{C}}: 18.27,21.33,24.69,86.76,92.58,100.09,112.89,126.56,126.69,129.77$, 129.84, 130.34, 138.76, 139.18, 140.45, 142.41, 150.08, 150.53, 154.39, 154.63, 154.96, 158.91; MS $(\mathrm{m} / \mathrm{z})$ : Calcd 461.20, found $461.28\left([\mathrm{M}+\mathrm{H}]^{+}\right)$; Anal. Calcd. for $\mathrm{C}_{28} \mathrm{H}_{24} \mathrm{~N}_{6} \mathrm{O}: \mathrm{C}, 73.02 ; \mathrm{H}$, $5.25 ; \mathrm{N}, 18.25 \%$. Found: C, 73.07; H, 5.28; N, 18.21\%.

5,5'-Dimethyl-2,2'-di(p-chlorophenyl)-4H-[6,7']bi(pyrazolo[1,5-a]pyrimidinyl)-7-one (3h). Yield $81 \%, \mathrm{mp}>330{ }^{\circ} \mathrm{C}$; IR $\left(v_{\max }, \mathrm{cm}^{-1}\right): 2962,1612,1582,1435 ;{ }^{1} \mathrm{H}$ NMR (DMSO, $\left.\mathrm{d}^{6}\right) \delta_{\mathrm{H}}: 2.22$ (s, 3H, 5- $\left.\mathrm{CH}_{3}\right), 2.60$ (s, 3H, 5'-CH $), 6.82$ (s, 1H, 6'-H), 7.08 (s, 1H, 3-H), 7.19 (s, 1H, 3'-H), 7.48 (d, 2 H, J 8.7 Hz, Ph-2", 6"-H), 7.56 (d, 2H, J 8.7Hz, Ph-2", 6"-H), 7.94 (d, 2H, J 8.7 Hz, Ph-3", 5"-H), 8.07 (d, 2H, J 8.7 Hz, Ph-3", 5"-H), 13.05 (s, 1H, NH, exchangeable with $\mathrm{D}_{2} \mathrm{O}$ ). ${ }^{13} \mathrm{C}$ NMR 
$\left(\mathrm{DMSO}, \mathrm{d}^{6}\right) \delta_{\mathrm{C}}: 18.35,24.75,79.59,87.20,93.13,100.10,113.29,128.35,128.53,129.27$, $129.33,131.47,132.02$, 133.85, 134.24, 140.48, 142.62, 150.12, 150.86, 153.18, 153.63, 154.52, 159.25; MS $(\mathrm{m} / \mathrm{z})$ : calcd 501.09, 503.09, 505.18 found 501.19, 503.18, $505.18(9: 6: 1)([\mathrm{M}+\mathrm{H}]$ $\left.{ }^{+} /[\mathrm{M}+\mathrm{H}+2]^{+} /[\mathrm{M}+\mathrm{H}+4]^{+}\right)$; Anal. Calcd. for $\mathrm{C}_{26} \mathrm{H}_{18} \mathrm{Cl}_{2} \mathrm{~N}_{6} \mathrm{O}: \mathrm{C}, 62.29 ; \mathrm{H}, 3.62 ; \mathrm{N}, 16.76 \%$. Found: C, 62.24; H, 3.67; N, 16.70\%.

5,5'-Dimethyl-2,2'-di( $p$-bromophenyl)-4H-[6,7']bi(pyrazolo[1,5-a]pyrimidinyl)-7-one (3i). Yield $88 \%$, mp $290{ }^{\circ} \mathrm{C}(\mathrm{d})$; IR $\left(v_{\max }, \mathrm{cm}^{-1}\right): 3001,1612,1582,1435 ;{ }^{1} \mathrm{H}$ NMR (DMSO, $\left.\mathrm{d}^{6}\right) \delta_{\mathrm{H}}$ : 2.22 (s, 3H, 5- $\left.\mathrm{CH}_{3}\right), 2.60$ (s, 3H, 5'- $\left.\mathrm{CH}_{3}\right), 6.80$ (s, 1H, 6'-H), 7.07 (s, 1H, 3-H), 7.17 (s, 1H, 3'-H), 7.61 (d, 2 H, J 8.4 Hz, Ph-2", 6"-H), 7.70 (d, 2H, J 8.4 Hz, Ph-2", 6"-H), 7.88 (d, 2H, J 8.4 Hz, Ph-3", 5"-H), 8.00 (d, 2H, J 8.4 Hz, Ph-3", 5"-H), 13.05 (s, 1H, NH, exchangeable with $\mathrm{D}_{2} \mathrm{O}$ ). ${ }^{13} \mathrm{C}$ NMR (DMSO, d $\mathrm{d}^{6}$ ) $\delta_{\mathrm{C}}: 18.35,24.78,79.64,87.18,93.15,100.13,113.30,122.50,127.70$, 128.62, 128.81, 131.85, 132.17, 132.23, 132.40, 132.51, 140.47, 142.59, 150.15, 150.81, 153.18, 159.20; MS (m/z): Calcd 588.99, 590.99, 592.99 (1:2:1), found 588.97, 590.97, 592.97 (1:2:1) $\left([\mathrm{M}+\mathrm{H}]^{+} /[\mathrm{M}+\mathrm{H}+2]^{+} /[\mathrm{M}+\mathrm{H}+4]^{+}\right)$; Anal. Calcd. for $\mathrm{C}_{26} \mathrm{H}_{18} \mathrm{Br}_{2} \mathrm{~N}_{6} \mathrm{O}: \mathrm{C}, 52.90 ; \mathrm{H}, 3.07 ; \mathrm{N}$, 14.24\%. Found: C, 52.94; H, 3.11; N, 14.22\%.

5,5'-Dimethyl-2,2'-di( $p$-fluorophenyl)-4H-[6,7']bi(pyrazolo[1,5-a]pyrimidinyl)-7-one (3j). Yield 84\%, mp $310{ }^{\circ} \mathrm{C}(\mathrm{d})$; IR $\left(v_{\max }, \mathrm{cm}^{-1}\right): 3124,3063,1659,1620,1574 ;{ }^{1} \mathrm{H}$ NMR (DMSO, $\mathrm{d}^{6}$ ) $\delta_{\mathrm{H}}: 2.18\left(\mathrm{~s}, 3 \mathrm{H}, 5-\mathrm{CH}_{3}\right), 2.58\left(\mathrm{~s}, 3 \mathrm{H}, 5^{\prime}-\mathrm{CH}_{3}\right), 6.71\left(\mathrm{~s}, 1 \mathrm{H}, 66^{\prime}-\mathrm{H}\right), 7.03(\mathrm{~s}, 1 \mathrm{H}, 3-\mathrm{H}), 7.09$ (s, 1H, 3'-H), 7.21- 7.33 (m, 4H, Ph-2", 6"-H), 7..92-7.96 (m, 2H, Ph-3", 5"-H), 7.99-8.08 (m, 2H, Ph3", 5"-H), 13.04 (s, 1H, NH, exchangeable with $\left.\mathrm{D}_{2} \mathrm{O}\right) .{ }^{13} \mathrm{C}$ NMR(DMSO, d $\left.\mathrm{d}^{6}\right) \delta_{\mathrm{H}}: 18.32,24.78$, 79.55, 87.23, 93.16, 100.10, 113.30, 122.62, 128.54, 128.96, 129.37, 131.47, 132.49, 133.56, $134.12,140.43,142.60,150.16,150.88,153.16,153.56,154.45,159.23 ; \mathrm{MS}(\mathrm{m} / \mathrm{z}):$ Calcd 468.15, found $469.53\left([\mathrm{M}+\mathrm{H}]^{+}\right)$; Anal. Calcd. for $\mathrm{C}_{26} \mathrm{H}_{18} \mathrm{~F}_{2} \mathrm{~N}_{6} \mathrm{O}: \mathrm{C}, 66.66 ; \mathrm{H}, 3.87 ; \mathrm{N}, 17.94 \%$. Found: C, 66.69; H, 3.89; N, 17.98\%.

5-Ethyl-5'-methyl-2,2'-diphenyl-4H-[6,7']bi(pyrazolo[1,5-a]pyrimidinyl)-7-one (3k). Yield $88 \%, \mathrm{mp}>330{ }^{\circ} \mathrm{C}$; IR $\left(v_{\max }, \mathrm{cm}^{-1}\right): 3742,3618,3163,3124,3070,2986,2885,2824,2361,1705$, 1659, 1620, 1574, 1520, 1443; ${ }^{1} \mathrm{H}$ NMR (DMSO, d $\left.\mathrm{d}^{6}\right) \delta_{\mathrm{H}}: 1.13\left(\mathrm{t}, 3 \mathrm{H}, J 7.5 \mathrm{~Hz}, \mathrm{CH}_{3}\right), 2.34(\mathrm{q}$, $2 \mathrm{H}, J 7.5 \mathrm{~Hz}, \mathrm{CH}_{2}$ ), 2.60 (s, 3H, 5'- $\left.\mathrm{CH}_{3}\right), 6.78$ (s, 1H, 6'-H), 7.07 (s, 1H, 3-H), 7.15 (s, 1H, 3'-H), 7.40-7.53 (m, 6H, 2Ph-3", 4", 5"-H), 7.92 (d, 2 H, J 6.6 Hz, Ph-2", 6"-H), 8.03 (d, 2H, J 6.6 Hz, Ph-2", 6"-H), 13.05 (s, 1H, NH, exchangeable with $\mathrm{D}_{2} \mathrm{O}$ ). ${ }^{13} \mathrm{C}$ NMR (DMSO, d $\left.\mathrm{d}^{6}\right) \delta_{\mathrm{C}}: 13.36$, 24.72, 25.56, 87.14, 92.97, 99.57, 113.04, 126.63, 126.79, 129.23, 129.29, 129.66, 132.55, 133.08, 140.46, 142.71, 150.07, 154.42, 154.89, 155.09, 159.22; MS (m/z): Calcd 447.19, found $447.27\left([\mathrm{M}+\mathrm{H}]^{+}\right)$; Anal. Calcd. for $\mathrm{C}_{27} \mathrm{H}_{22} \mathrm{~N}_{6} \mathrm{O}: \mathrm{C}, 72.63 ; \mathrm{H}, 4.97 ; \mathrm{N}, 18.82 \%$. Found: C, 72.68; $\mathrm{H}, 4.94 ; \mathrm{N}, 18.78 \%$.

\section{5-Ethyl-5'-methyl-2,2'-di(p-methylphenyl)-4H-[6,7']bi(pyrazolo[1,5-a]pyrimidinyl)-7-one} (31). Yield 88\%, $\mathrm{mp}>330{ }^{\circ} \mathrm{C}$; IR $\left(v_{\max }, \mathrm{cm}^{-1}\right)$ : 3132, 3055, 3024, 2970, 2924, 2854, 1744, 1659, 1620, 1566, 1497, 1443; ${ }^{1} \mathrm{H}$ NMR (DMSO, d $\left.{ }^{6}\right) \delta_{\mathrm{H}}: 1.13\left(\mathrm{t}, 3 \mathrm{H}, J 7.5 \mathrm{~Hz}, \mathrm{CH}_{3}\right), 2.31\left(\mathrm{~s}, 3 \mathrm{H}, \mathrm{CH}_{3}\right)$, 2.36 (q, 2H, J $\left.7.5 \mathrm{~Hz}, \mathrm{CH}_{2}\right), 2.37$ (s, 3H, CH ), 2.59 (s, 3H, 5'- $\left.\mathrm{CH}_{3}\right), 6.73$ (s, 1H, 6'-H), 7.03 (s, 1H, 3-H), 7.10 (s, 1H, 3'-H), 7.22 (d, 2 H, J 7.8 Hz, Ph-2", 6"-H), 7.31 (d, 2H, J 7.8 Hz, Ph-2", 6"-H), 7.79 (d, 2H, J 7.8 Hz, Ph-3", 5"-H), 7.92 (d, 2H, J 7.8 Hz, Ph-3", 5"-H), 13.03 (s, 1H, NH, 
exchangeable with $\mathrm{D}_{2} \mathrm{O}$ ). ${ }^{13} \mathrm{C}$ NMR (DMSO, $\mathrm{d}^{6}$ ) $\delta_{\mathrm{C}}: 13.39,21.35,24.73,25.54,86.89,92.64$, $99.58,112.85,126.56,126.73,129.78,138.75,139.17,140.43,154.43,154.94,159.03$; MS $(\mathrm{m} / z)$ : Calcd 475.22, found $475.29\left([\mathrm{M}+\mathrm{H}]^{+}\right)$; Anal. Calcd. for $\mathrm{C}_{29} \mathrm{H}_{26} \mathrm{~N}_{6} \mathrm{O}: \mathrm{C}, 73.40 ; \mathrm{H}, 5.52 ; \mathrm{N}$, $17.71 \%$. Found: C, 73.46; H, 5.48; N, 17.75\%.

\section{5-Ethyl-5'-methyl-2,2'-di( $\boldsymbol{p}$-chlorophenyl)-4H-[6,7']bi(pyrazolo[1,5-a]pyrimidinyl)-7-one} (3m). Yield 88\%, mp > $320{ }^{\circ} \mathrm{C}$; IR $\left(v_{\max }, \mathrm{cm}^{-1}\right): 3742,3649,3618,3124,3055,2978,2885$, 2831, 2361, 1774, 1651, 1620, 1558, 1520, 1443; ${ }^{1} \mathrm{H}$ NMR (DMSO, d $\left.{ }^{6}\right) \delta_{\mathrm{H}}: 1.15$ (t, 3H, J 7.2 Hz, $\mathrm{CH}_{3}$ ), 2.36 (q, 2H, J $7.2 \mathrm{~Hz}, \mathrm{CH}_{2}$ ), 2.61 (s, 3H, 5'- $\mathrm{CH}_{3}$ ), 6.81 (s, 1H, 6'-H), 7.09 (s, 1H, 3-H), 7.19 (s, 1H, 3'-H), 7.48 (d, 2 H, J 8.4 Hz, Ph-2", 6"-H), 7.57 (d, 2H, J 8.4 Hz, Ph-2", 6"-H), 7.93 (d, 2H, J 8.4 Hz, Ph-3", 5"-H), 8.07 (d, 2H, J 8.4 Hz, Ph-3", 5"-H), 13.12 (s, 1H, NH, exchangeable with $\mathrm{D}_{2} \mathrm{O}$ ). ${ }^{13} \mathrm{C}$ NMR (DMSO, $\mathrm{d}^{6}$ ) $\delta_{\mathrm{C}}$ : 13.38, 24.76, 25.56, 87.31, 93.21, 99.63, 113.24, 128.34, 128.56, 129.26, 129.32, 131.52, 132.04, 133.85, 134.26, 140.44, 142.83, 150.15, 153.67, 154.73, 155.19, 159.36; MS (m/z): calcd 515.11, 517.11, 519.11 found 515.20, 517.19, $519.19(9: 6: 1)\left([\mathrm{M}+\mathrm{H}]^{+} /[\mathrm{M}+\mathrm{H}+2]^{+} /[\mathrm{M}+\mathrm{H}+4]^{+}\right)$; Anal. Calcd. for $\mathrm{C}_{27} \mathrm{H}_{20} \mathrm{Cl}_{2} \mathrm{~N}_{6} \mathrm{O}: \mathrm{C}, 62.92 ; \mathrm{H}$, $3.91 ; \mathrm{N}, 16.31 \%$. Found: C, 62.88; H, 3.92; N, 16.33\%.

\section{5-Ethyl-5'-methyl-2,2'-di(p-bromophenyl)-4H-[6,7']bi(pyrazolo[1,5-a]pyrimidinyl)-7-one} (3n). Yield 84\%, mp $300{ }^{\circ} \mathrm{C}$ (d); IR ( $\left.v_{\max }, \mathrm{cm}^{-1}\right): 3186,3132,3078,2924,2854,1674,1620$, 1558, 1520, 1435; ${ }^{1} \mathrm{H}$ NMR (DMSO, d $\left.\mathrm{d}^{6}\right) \delta_{\mathrm{H}}: 1.12\left(\mathrm{t}, 3 \mathrm{H}, J 7.2 \mathrm{~Hz}, \mathrm{CH}_{3}\right.$ ), 2.35 (q, 2H, $J .2 \mathrm{~Hz}$, $\mathrm{CH}_{2}$ ), 2.61(s, 3H, 5'-CH - $_{3}, 6.80$ (s, 1H, 6'-H), 7.08 (s, 1H, 3-H), 7.18 (s, 1H, 3'-H), 7.61 (d, 2 H, J $8.1 \mathrm{~Hz}, \mathrm{Ph}-2 ", 6$ "-H), 7.69 (d, 2H, J 8.1 Hz, Ph-2", 6"-H), 7.86 (d, 2H, J 8.1 Hz, Ph-3", 5"-H), $8.00(\mathrm{~d}, 2 \mathrm{H}, J 8.1 \mathrm{~Hz}, \mathrm{Ph}-3 ", 5 "-\mathrm{H}), 13.07$ (s, 1H, NH, exchangeable with $\left.\mathrm{D}_{2} \mathrm{O}\right) .{ }^{13} \mathrm{C}$ NMR $\left(\mathrm{DMSO}, \mathrm{d}^{6}\right) \delta_{\mathrm{C}}: 13.38,24.76,25.58,87.30,99.62,113.27,122.92,128.61,128.82,132.22$, $140.44,142.82,150.16,153.31,154.75,155.20,159.32,159.37 . \mathrm{MS}(\mathrm{m} / \mathrm{z}):$ Calcd 603.00, $605.00,607.00(1: 2: 1)$, found $603.14,605.14,607.13(1: 2: 1)\left([\mathrm{M}+\mathrm{H}]^{+} /[\mathrm{M}+\mathrm{H}+2]^{+} /[\mathrm{M}+\mathrm{H}+4]^{+}\right)$; Anal. Calcd. for $\mathrm{C}_{27} \mathrm{H}_{20} \mathrm{Br}_{2} \mathrm{~N}_{6} \mathrm{O}: \mathrm{C}, 53.66 ; \mathrm{H}, 3.34 ; \mathrm{N}, 13.91 \%$. Found: $\mathrm{C}, 53.62 ; \mathrm{H}, 3.42 ; \mathrm{N}$, $13.94 \%$.

\section{5-Ethyl-5'-methyl-2,2'-di( $\boldsymbol{p}$-fluorophenyl)-4H-[6,7']bi(pyrazolo[1,5-a]pyrimidinyl)-7-one}

(3o). Yield 87\%, mp $290{ }^{\circ} \mathrm{C}(\mathrm{d})$; IR $\left(v_{\max }, \mathrm{cm}^{-1}\right)$ : 3124, 3055, 2978, 1612, 1528, 1443; ${ }^{1} \mathrm{H}$ NMR (DMSO, d $\left.{ }^{6}\right) \delta_{\mathrm{H}}: 1.12\left(\mathrm{t}, 3 \mathrm{H}, J 7.2 \mathrm{~Hz}, \mathrm{CH}_{3}\right), 2.37\left(\mathrm{q}, 2 \mathrm{H}, J 7.2 \mathrm{~Hz}, \mathrm{CH}_{2}\right), 2.60\left(\mathrm{~s}, 3 \mathrm{H}, 5^{\prime}-\mathrm{CH}_{3}\right)$, 6.77 (s, 1H, 6'-H), 7.07 (s, 1H, 3-H), 7.14 (s, 1H, 3'-H), 7.22-7.27 (m, 2 H, Ph-2", 6"-H), 7.7.307.36 (m, 2H, Ph-2", 6"-H), 7.93-7.97 (m, 2H, Ph-3", 5"-H), 8.06-8.11 (m, 2H, Ph-3", 5"-H), $13.04\left(\mathrm{~s}, 1 \mathrm{H}, \mathrm{NH}\right.$, exchangeable with $\left.\mathrm{D}_{2} \mathrm{O}\right) .{ }^{13} \mathrm{C}$ NMR (DMSO, $\mathrm{d}^{6}$ ) $\delta_{\mathrm{C}}: 13.37,24.76,25.55$, 87.30, 92.92, 113.22, 116.04, 116.27, 128.82, 128.94, 140.45, 142.79, 150.16, 153.49, 153.94, 154.76, 155.07, 159.24; MS $(\mathrm{m} / \mathrm{z})$ : Calcd 483.17, found $483.56\left([\mathrm{M}+\mathrm{H}]^{+}\right)$; Anal. Calcd. for $\mathrm{C}_{27} \mathrm{H}_{20} \mathrm{~F}_{2} \mathrm{~N}_{6} \mathrm{O}: \mathrm{C}, 67.21 ; \mathrm{H}, 4.18 ; \mathrm{N}, 17.42 \%$. Found: $\mathrm{C}, 67.25 ; \mathrm{H}, 4.15 ; \mathrm{N}, 17.40 \%$.

Evaluation of antibacterial activity. For the evaluation of antibacterial activity of the compounds, the size of inoculum was adjusted to approximately $10^{8}$ colony-forming units $\mathrm{cfu} / \mathrm{mL}$ by suspending the culture in sterile distilled water. Petri dishes containing $25 \mathrm{~mL}$ of nutrient agar medium were swabbed with $100 \mu \mathrm{L}$ culture of the respective bacterial strains and kept for $15 \mathrm{~min}$ for the absorption of culture. Using a sterile cork borer, $\sim 8 \mathrm{~mm}$ diameter well was 
bored in the seeded agar plates and a $100 \mu \mathrm{L}$ volume of each test compound of concentration 10 , $50,100 \mu \mathrm{g} / \mathrm{mL}$, reconstituted in DMSO was added into the wells. DMSO was used as control for all the test compounds. All the plates were incubated at $37{ }^{\circ} \mathrm{C}$ for $24 \mathrm{~h}$. Antibacterial activity was determined by measuring the zone of inhibition around the well.

Determination of minimum inhibitory concentration (MIC). MIC of all the compounds was determined by the agar well diffusion method. Petri dishes containing $25 \mathrm{~mL}$ nutrient agar medium was swabbed with the $100 \mu \mathrm{L}$ culture of inoculum size of approximately $10^{8}$ colonyforming units $\mathrm{cfu} / \mathrm{ml}$. Twofold serial dilution ranging from $200-0.19$ of chemically synthesised compound were made. Dilutions were made in DMSO. Using a sterile cork borer, $\sim 8 \mathrm{~mm}$ diameter well was bored in the seeded agar plates and a $100 \mu \mathrm{L}$ volume of different dilutions of each test compound was added into the wells. These plates were incubated at $37^{\circ} \mathrm{C}$ for $24 \mathrm{~h}$.

\section{Acknowledgements}

We thank the council of Scientific and Industrial Research, New Delhi for financial assistance and Senior Research Fellowship to Chinu Rani. Thanks are also due to the Mass spectrometry Facility, University of California, San Francisco, USA for running the mass spectra.

\section{References:}

1. Arratt, S. J. Org. Chem. 1963, 28, 1886. http://dx.doi.org/10.1021/jo01042a038

2. Stephen, J. F.; Marcus, E. J. Org. Chem. 1969, 34, 2527. http://dx.doi.org/10.1021/jo01261a011

3. Gelin, S.; Chantegral, B.; Nadi, A.; Fruchier, A. J. Org. Chem. 1983, 48, 4078. http://dx.doi.org/10.1021/jo00170a041

4. Singh, S. P.; Prakash O.; Vaid, R. K. Indian J. Chem. Sect. B 1984, 23, 191.

5. Kumar D.; Singh, S. P. J. Indian Chem. Soc. 2006, 83, 419.

6. Inoue, A.; Iguchi, S. Chem. Pharm. Bull. 1964, 12, 381. http://dx.doi.org/10.1248/cpb.12.381

7. Sanz, D.; Claramunt, R. M.; Saini, A.; Kumar, V.; Aggarwal, R.; Singh, S. P.; Alkorta I.; Elguero J. Reson. Chem Magn.2007, 45, 513.

http://dx.doi.org/10.1002/mrc.1992 PMid:17405108

8. Shi, C.-L.; Shi, D.-Q.; Kim, S. H.; Huang, Z.-B.; Ji, S.-J.; Ji, M. Tetrahedron 2008, 64, 2425. http://dx.doi.org/10.1016/j.tet.2007.12.053

9. An, H.; Eum, S. J.; Koh, M.; Lee, S.K.; Park, S. B. J. Org. Chem. 2008, 73, 1752. http://dx.doi.org/10.1021/jo702196f 
PMid:18266382

10. Kiessling, A.; Wiesinger, R.; Sperl, B.; Berg, T. ChemMedChem 2007, 2, 627.

http://dx.doi.org/10.1002/cmdc.200600294

PMid:17315254

11. Aggarwal, R.; Kumar, V.; Bansal, A.; Sanz, D.; Claramunt, R. M. J. Fluorine Chem. 2012, 140,31 .

http://dx.doi.org/10.1016/j.jfluchem.2012.04.007

12. Gregg, B. T.; Tymoshenko, D. O.; Razzano, D. A.; Johnson, M. R. J. Comb. Chem. 2007, 9, 507.

http://dx.doi.org/10.1021/cc0700039

PMid:17439287

13. Selleri, S.; Bruni, F.; Costagli, C.; Costanzo, A.; Guerrini, G.; Ciciani, G.; Gratteri, P.; Besnard, F.; Costa, B.; Montali, M.; Martini, C.; Fohlin, J.; De Siena, G.; Aiello, P. A. J. Med. Chem. 2005, 48, 6756.

http://dx.doi.org/10.1021/jm058002n

PMid:16220991

14. Mason, J. S.; Morize, I.; Menard, P. R.; Cheney, D. L.; Hulme, C.; Labaudiniere, R. F. J. Med. Chem. 1995, 42, 3251.

http://dx.doi.org/10.1021/jm9806998

PMid:10464012

15. Novinson, T.; Hanson, R.; Dimmitt, M. K.; Simon, L. N.; Robins, R. K.; Obrien, D. E. J. Med. Chem. 1974, 17, 645.

http://dx.doi.org/10.1021/jm00252a016

16. Novinson, T.; Miller, J. P.; Scholten, M.; Robins, R. K.; Simon, L. N.; Obrien, D. E.; Meyer, R. B. J. Med. Chem. 1975, 18, 460. http://dx.doi.org/10.1021/jm00239a004

17. Makarov, V. A.; Solov'eva, N. P.; Granlk, V. G. Chem. Heterocycl. Compds. 1997, 33, 535. http://dx.doi.org/10.1007/BF02291935

18. Kirkpatrick, W. E.; Okabe, T.; Hillyard, I. W.; Robins, R. K.; Dren, A.T.; Novinson, T. J. Med. Chem. 1977, 20, 386. http://dx.doi.org/10.1021/jm00213a014

19. Aggarwal, R.; Kumar, R.; Kumar, S.; Garg, G.; Mahajan, R.; Sharma, J. J. Fluorine Chem. 2011, 132, 965. http://dx.doi.org/10.1016/j.jfluchem.2011.07.029

20. Aggarwal, R.; Kumar, V.; Kumar, R.; Singh, S. P. Beilstein J. Org. Chem. 2011, 7, 179. http://dx.doi.org/10.3762/bjoc.7.25 PMid:21448263 PMCid:3063075

21. Singh, S. P.; Naithani, R.; Aggarwal, R.; Prakash, O. Synth. Commun. 2004, 34, 4359. http://dx.doi.org/10.1081/SCC-200039427 
22. Aggarwal, R.; Sumran, G.; Claramunt, R. M.; Sanz, D.; Elguero, J. J. Mol. Str. 2009, 934, 96. http://dx.doi.org/10.1016/j.molstruc.2009.06.021

23. Aggarwal, R.; Sumran, G.; Garg, N.; Aggarwal, A. Eur. J. Med. Chem. 2011, 46, 3038. http://dx.doi.org/10.1016/j.ejmech.2011.04.041 PMid:21558044

24. Aggarwal, R.; Sumran, G.; Kumar, V.; Mittal, A. Eur. J. Med. Chem. 2011, 46, 6083. http://dx.doi.org/10.1016/j.ejmech.2011.10.032 PMid:22051064

25. Bebehani, H.; Ibrahim, H. M.; Makhseed, S. Arkivoc 2010, (ii), 267-282. http://dx.doi.org/10.3998/ark.5550190.0011.222

26. Bassoude, I.; Berteina-Raboin, S.; Leger, J-M.; Jarry, C.; Essassi, E. M.; Guillaumet, G. Tetrahedron 2011, 67, 2279. http://dx.doi.org/10.1016/j.tet.2011.01.070

27. Nam, N. L.; Grandberg, I. I.; Sorokin. V. I. Chem. Heterocycl. Compd. 2002, 38, 1371. http://dx.doi.org/10.1023/A:1022186627777

28. Petrov, A. A.; Emelina, E. E.; Selivanov, S. I. Russ. J. Org. Chem. 2008, 44, 263. http://dx.doi.org/10.1134/S1070428008020139

29. Makarov, V. A.; Riabova, O. B.; Granik, V. G.; Dahse, H.; Stelzner, A.; Wutzler, P.; Schmidtke M. Bioorg. Med. Chem. Lett. 2005, 15, 37.

http://dx.doi.org/10.1016/j.bmcl.2004.10.043

PMid:15582406

30. Petrova, O. V.; Sobenina, L. N.; Demenev, A. P.; Mikhaleva, A. I.; Ushakov, I. A. Russ. J. Org. Chem. 2003, 39, 1471. http://dx.doi.org/10.1023/B:RUJO.0000010564.39518.5e

31. Ahmad, I.; Beg, A. Z. J. Ethnopharmacology 2001, 74, 113. http://dx.doi.org/10.1016/S0378-8741(00)00335-4

32. Andrews, J. M. J. Antimicrobial Chemotherapy 2001, 48, 5. http://dx.doi.org/10.1093/jac/48.suppl 1.5 